\title{
Neuronal loss in primary long-term cortical culture involves neurodegeneration-like cell death via calpain and p35 processing, but not developmental apoptosis or aging
}

\author{
Min-Ju Kim ${ }^{1}$, Soo-Jin $\mathrm{Oh}^{1}$, Seong-Hoon Park ${ }^{1}$, \\ Hong-Jun Kang ${ }^{1}$, Moo Ho Won ${ }^{2}$, \\ Tae-Cheon Kang', Jae-Bong Park', Jong-ll Kim', \\ Jaebong $\mathrm{Kim}^{1}$ and Jae-Yong Lee ${ }^{1,3}$ \\ ${ }^{1}$ Department of Biochemistry \\ ${ }^{2}$ Department of Anatomy \\ College of Medicine, Hallym University \\ Chuncheon 200-702, Korea \\ ${ }^{3}$ Corresponding author: Tel, 82-33-248-2543; \\ Fax, 82-33-244-8425; E-mail, jyolee@hallym.ac.kr
}

Accepted 28 November 2006

Abbreviations: ALLM, N-Acetyl-Lue-Lue-Norleu-al; ALLN, N-AcetylLeu-Leu-Met-al; Ara-C, cytosine arabinoside; GFAP, glial-fibrillary acidic protein; PARP, poly (ADP ribose) polymerase; zVAD-fmk, $\mathrm{N}$-benzyloxycarbonyl-Val-Ala-Asp-fluoromethylketone

\begin{abstract}
Primary neuronal culture is a powerful tool to study neuronal development, aging, and degeneration. However, cultured neurons show signs of cell death after 2 or 3 weeks. Although the mechanism underlying this phenomenon has not been elucidated, several preventive methods have been identified. Here we show that the neuronal loss in primary cortical culture involves calpain activation and subsequent neuronal cell death. Neuronal loss during cultivation showed destruction of neurites and synapses, and a decrease in neuron numbers. $\mu$-Calpain and $m$-calpain were initially activated and accumulated by increased RNA expression. This neuronal death exhibited neurodegenerative features, such as conversion of p35 to p25, which is important in the developmental process and in the pathogenesis of Alzheimer's disease. But, postnatal and aged rat cortex did not show calpain activation and prolonged processing of $\mathrm{p} 35$ to $\mathrm{p} 25$, in contrast to the long-term culture of cortical neurons. In addition, the inhibition of calpains by ALLM or ALLN blocked the conversion of $\mathrm{p} 35$ to $\mathrm{p} 25$, indicating that the calpain activity is essential for the neurodegenerative features of cell death. Taken together, this
\end{abstract}

study shows that the neuronal loss in primary cortical cultures involves neurodegeneration-like cell death through the activation of calpains and the subsequent processing of $p 35$ to $p 25$, but not developmental apoptosis or aging. Our results suggest that the long term primary culture of cortical neurons represent a valuable model of neurodegeneration, such as Alzheimer's disease.

Keywords: Alzheimer disease; calpain; cells, cultured; cell death; nerve degeneration

\section{Introduction}

The central nervous system (CNS) is vulnerable to defects, diseases, and injuries throughout development and adulthood. In many abnormal CNS conditions, neurons in the cerebral cortex are highly vulnerable. In immature and mature brains, cortical neurons degenerate after hypoxia-ischemia, trauma, and seizure, and a subset of neocortical neurons degenerate in Alzheimer's disease, amyotropic lateral sclerosis, Parkinson's disease, and Huntington's disease (Morrison and Hof, 1997). Despite many advances in the understanding of the mechanisms that cause abnormalities during neuronal development and a loss of neuronal viability, no effective restorative or therapeutic treatments exist for most developmental and for all neurodegenerative disorders.

Many in vivo and in vitro studies have been performed on neuronal dysfunction and degeneration. These systems have particular advantages and limitations. First, animal model systems can be used to reveal many aspects of neuronal development and neuronal degeneration and provide physiological meaning directly (Martin et al., 1998a). However, definitive comprehension of molecular, biochemical, and structural observations is often obscured, due to the inherent complexity of nervous system. Compared with in vivo systems, in vitro cultures provide homogeneous and uniform data (Martin et al., 1998b). Furthermore, neuronal development and degeneration should be considered not as separate topics, but as a continuum (Castagne et al., 1999). Thus, many developmental or degenerative disorders even including aging-associated change, have been studied 
in vivo and in vitro (Aguzzi et al., 1996; Silani et al., 2000).

Banker and Cowan first developed an in vitro system for the study of isolated hippocampal neurons from 18 day old fetuses of rats (1977). Cells in these original cultures were cocultured with tissues or hippocampal explants and survived only for 2 weeks. Since these initial studies, in vitro systems for isolated hippocampal and cortical neurons and neuronal cell lines have gradually improved, and are used widely in various studies (Ray et al., 1993). For example, serum-free combination media, such as B27-supplemented neurobasal medium, has shown an excellent ability for long term survival of neurons (Brewer et al., 1993; Evans et al., 1998). However, neurobasal medium contains vitamin $\mathrm{E}$, glutathione, catalase, superoxide dismutase, and transferrin, which increase neuronal resistance to biological oxidants, and thus it is not recommended for survival studies, e.g. damages by free radicals or oxidation (Xie et al., 2000). So far, the studies on short survival of cultured neuronal cells have not identifed the mechanistics involved or the association with normal brain aging or the pathogenesis or disease courses of neurodegenerative diseases such as Alzheimer's disease, Parkinson disease, or ischemic dementia.

Calpains are $\mathrm{Ca}^{2+}$-dependent proteases that mediate various physiological and pathological functions. The two major calpain family proteins, $\mu$-calpain and $\mathrm{m}$-calpain, share a $30 \mathrm{kDa}$ regulatory subunit and require different $\mathrm{Ca}^{2+}$ concentrations for activation in vitro. Activated calpain acts on a host of endogenous proteins, which include cytoskeletal proteins (fodrin, vimentin, keratin, and tau), PARP, calmodulin-dependent protein kinase IV (CaMKIV), clathrin assembly protein, bax, and even procaspase-3 (Ando et al., 1988; Litersky and Johnson, 1995; McGinnis et al., 1998; Prasad et al., 1998; McGinnis et al., 1999a; Kim and Kim, 2001). Particularly, in many neurodegenerative disorders such as Alzheimer's disease, this calpain systems are involved (Patrick et al., 1999; Lee et al., 2000). In Alzheimer's disease, calpain is activated by amyloid-beta peptides and resultant activated calpains cleave p35 protein into p25. Then, p25 activates CDK5 that would lead to pathologic phosphorylation of tau proteins. p35 is known to mediate various physiological phenomena involving brain development with CDK5 (Nguyen et al., 2002). Recent data suggest that calpain activation occurs in normal aging as well as neurodegenerative disorders (Czogalla and Sikorski, 2005).

Initially, we detected calpain and p35 processing in long-term culture of primary cortical neurons. To understand these activations and neuronal loss during long-term cultivation, we explored the features of neuronal development, aging and degeneration.

\section{Materials and Methods}

\section{Materials}

Calpain inhibitors, N-Acetyl-Lue-Lue-Norleu-al (ALLM) and N-Acetyl-Leu-Leu-Met-al (ALLN), were from Calbiochem. N-benzyloxycarbonyl-Val-Ala-Asp-fluoromethylketone (zVAD-fmk), broad range caspase inhibitor, was obtained from PharMingen. Chemicals for electrophoresis were purchased from Bio-Rad and Fisher Scientific. Other materials were obtained from Sigma Chemicals.

\section{Cell culture}

Primary neuronal cultures were prepared from the cerebral cortex of embryonic day 18 rat embryos. Briefly, the cortices of removed embryos were dissected and freed of meninges. The cells were dissociated by trypsinization $(0.25 \%$ for $15 \mathrm{~min}$ at $37^{\circ} \mathrm{C}$ ), followed by trituration with a fire-polished Pasteur pipette and plated onto poly-D-lysine-coated $60 \mathrm{~mm}$ dishs containing DMEM with $5 \%$ fetal bovine serum and $5 \%$ horse serum. Non-neuronal cell division was arrested after $48 \mathrm{~h}$ in $10 \mu \mathrm{M}$ cytosine arabinoside (Ara-C) and the media was changed after $24 \mathrm{~h}$. Cultures were maintained at $37^{\circ} \mathrm{C}$ in a humidified incubator containing $5 \% \mathrm{CO}_{2}$ and culture media were changed every 3 days. Some of cortical cells were treated with $1 \%$ serum (10 times dilution of DMEM with $5 \%$ horse serum and $5 \%$ fetal bovine serum) and $5 \mu \mathrm{M}$ cytosine arabinoside every 5 days intermittently to suppress the growth of non-neuronal cells.

\section{Western blotting}

For whole cell lysate preparation, cells were washed twice with ice-cold PBS and resuspended in a buffer containing $150 \mathrm{mM} \mathrm{NaCl}, 50 \mathrm{mM}$ EDTA, $0.5 \mathrm{M}$ Tris- $\mathrm{HCl}(\mathrm{pH} 7.4), 1 \% \mathrm{NP} 40,4 \mathrm{mM}$ phenylmethylsulfonylfluoride(PMSF), $1 \mu \mathrm{M}$ aprotinin, and $1 \mu \mathrm{M}$ leupeptin. The extracts were vortexed and centrifuged at $17,000 \times \mathrm{g}$ for $20 \mathrm{~min}$ at $4^{\circ} \mathrm{C}$. The clarified supernatants were stored at $-70^{\circ} \mathrm{C}$, and protein concentration was measured using the Lowry assay (Biorad). The proteins were resolved by SDS-PAGE and elctroblotted onto a PVDF membrane (Millipore, Bedford, MA). The following monoclonal mouse antibodies were used; anti-synaptophysin $(1: 1,000$, Santa Cruz Biotechnology), anti-syntaxin (1:1,000, Santa Cruz Biotechnology), anti-actin (1:1,000, Santa Cruz Biotechnology), anti-bax (1:500, Transduction Laboratory), anti-bcl-2 (1:1,000, Transduction Laboratory), anti-PARP $(1: 1,000$, Pharmingen), anti- $\mu$-calpain $(1: 2,000$, Chemicon), anti-m-calpain $(1: 1,000$, 
Biomol), anti-NeuN (1:500, Chemicon), and polyclonal rabbit antibodies; anti-p35 $(1: 1,000$, Santa Cruz Biotechnology), anti-caspase-3 (1:1,000, Santa Cruz Biotechnology). Secondary antibodies conjugated to horse radish peroxidase $(1: 5,000$, Pierce) followed by enhanced chemiluminescence reagents (Amersham) were used for the detection of peroxidase.

\section{mRNA isolation}

Total RNA was obtained using Trizol reagent (Gibco) following manufacturer's protocol. Cortical cells and brain tissues at appropriate time were collected with RNase free PBS. Total mRNA was extracted with Trizol and chloroform, precipitated with isopropanol, and then dissolved in RNA free water. RNA concentration was determined spectrophotometrically.

\section{Reverse transcription-polymerase chain reaction}

$1 \mu \mathrm{g}$ of mRNA was used for each reverse transcriptase (RT) reaction. RT was performed with oligodT methods. Briefly, complementary DNA (cDNA) was generated by incubating $1 \mu \mathrm{g}$ of mRNA with oligo-dT (200 mg/ml), deoxyribonucleotide (dNTPs, $10 \mathrm{mM}$ ), $1 \times$ first strand buffer and $5 \mathrm{U}$ of AMV reverse transcriptase (Takara, Japan) at $42^{\circ} \mathrm{C}$ for $2 \mathrm{~h}$ and then incubated at $72^{\circ} \mathrm{C}$ for $15 \mathrm{~min}$. The resultant cDNA was used directly in the PCR, which was performed as follows. cDNA samples were mixed with dNTPs (10 mM), individual forward and reverse primers (10 pmol), $1 \times \mathrm{PCR}$ buffer (containing 1.5 $\left.\mathrm{mM} \mathrm{MgCl}_{2}, \mathrm{pH} 10.0\right)$ and Taq DNA polymerase (5
$\mathrm{U} / \mu \mathrm{l}$; Biotools, Spain) in $25 \mu \mathrm{l}$ reaction volume. The details for the primers are shown in Table 1. PCR reactions were carried out using Robocycler (Stratagene) as follows; $94^{\circ} \mathrm{C}$ for $5 \mathrm{~min}, 28 / 33$ cycles of $94^{\circ} \mathrm{C}$ for $1 \mathrm{~min}, 55 / 58^{\circ} \mathrm{C}$ for $1 \mathrm{~min}$, and $72^{\circ} \mathrm{C}$ for 1 $\mathrm{min}$, and an extension at $72^{\circ} \mathrm{C}$ for $10 \mathrm{~min}$. The PCR products were separated electrophoretically on $1.2 \%$ agarose gel with $1 \mu \mathrm{g} / \mathrm{ml}$ ethidium bromide.

\section{Terminal deoxynucleotidyl transferase-mediated dUTP nick-end labeling (TUNEL) assay}

A TUNEL assay was performed to demonstrate DNA fragmentation in single cells, which occurs during apoptosis. DNA strand breaks were identified by labeling free 3 '-OH termini with modified dUTP in an enzymatic reaction using TdT. Briefly, cortical cells, grown on cover glass for 15 days, were fixed with $4 \%$ paraformaldehyde in phosphate-buffered saline (PBS) for $15 \mathrm{~min}$ and permeabilized with $0.2 \%$ Triton $\mathrm{X}-100$ in PBS containing $1 \%$ bovine serum albumin for $15 \mathrm{~min}$. Cells were rinsed in TdT buffer $(25-\mathrm{mM}$ Tris $\mathrm{HCl}, 200 \mathrm{mM}$ sodium cacodylate, $2.5 \mathrm{mM}$ cobalt chloride) and incubated with the labeling mix (20 U of TdT, Boehringer Mannheim) and $1 \mathrm{nmol}$ of Digoxigenen-11-dUTP (Boehringer Mannheim) in $100 \mu \mathrm{l}$ of TdT buffer for $2 \mathrm{~h}$ at $37^{\circ} \mathrm{C}$. The reaction was stopped by rinsing in $300 \mathrm{mM}$ sodium chloride/30 $\mathrm{mM}$ sodium citrate, and placed in Tris-buffered saline (TBS) containing $0.3 \%$ Triton $X-100$ and $2 \%$ bovine serum albumin for $15 \mathrm{~min}$ at room temperature. They were incubated with anti-digoxigenin sheep antibody conjugated with alkaline phosphatase (Boehringer Mannheim), diluted 1:250 in TBS-

Table 1. Primer sequences for RT-PCR experiments.

\begin{tabular}{|c|c|c|c|c|}
\hline Primer & Sequence & Product size & Cycle (Tm) & Reference \\
\hline caspase-3 & $\begin{array}{l}\text { F:GCCGACTTCCTGTATGCTTA } \\
\text { R:GCAGGCAGTGGTATTGTTCA }\end{array}$ & 602 bp & $\begin{array}{c}* * 31 / 33 \\
\left(55^{\circ} \mathrm{C}\right)\end{array}$ & (Ecarnot-Laubriet et al., 2002) \\
\hline$\mu$-calpain & $\begin{array}{l}\text { F:CACTTGAAGCGTGACTTCTTCCTGGCCAATGC } \\
\text { R:GCACTCATGCTGCCCGACTTGTCCAGGTCAAACTT }\end{array}$ & 536 bp & $\begin{array}{c}* * 27 / 29 \\
\left(58^{\circ} \mathrm{C}\right)\end{array}$ & (Ray et al., 2000) \\
\hline m-calpain & $\begin{array}{l}\text { F:GGGCAGACCAACATCCACCTCAGCAAAAAC } \\
\text { R:GTCTCGATGCTGAAGCCATCTGACTTGAT }\end{array}$ & 404 bp & $\begin{array}{l}* * 28 / 30 \\
\left(58^{\circ} \mathrm{C}\right)\end{array}$ & (Ray et al., 2000) \\
\hline calpastatin & $\begin{array}{l}\text { F:AGTAGTTCTGGACCCAATG } \\
\text { R:CCCCAGTAGACTTCTCTTTC }\end{array}$ & $230 \mathrm{bp}$ & $\begin{array}{c}30 \\
\left(58^{\circ} \mathrm{C}\right)\end{array}$ & (Ray et al., 2000) \\
\hline GFAP & $\begin{array}{l}\text { F:GCTGGAGGTGGAGAGGGACA } \\
\text { R:TGGCGGCGATAGTCTTTAGC }\end{array}$ & 456 bp & $\begin{aligned} * * 28 / 30 \\
\left(56^{\circ} \mathrm{C}\right)\end{aligned}$ & (Crispino et al., 2001) \\
\hline actin & $\begin{array}{l}\text { F:TGGAATCCTGTGGCATCCATGAAAC } \\
\text { R:TAAAACGCAGCTCAGTAACAGTCCG }\end{array}$ & 348 bp & $\begin{array}{c}30 \\
\left(55^{\circ} \mathrm{C}\right)\end{array}$ & (Faulkner et al., 1995) \\
\hline
\end{tabular}

${ }^{*} \mathrm{~F}$, forward; $\mathrm{R}$, reverse; Tm, annealing temperature; Cycle, number of PCR cycles.

${ }^{* *}$ In Figure 4B, we performed RT-PCR with lower PCR-cycles to examine the differences between $1 \%$ Ara-C and low-serum treat at DIV15. 
Triton $\mathrm{X}-100$ for $30 \mathrm{~min}$ at $37^{\circ} \mathrm{C}$. The color reaction was revealed with $50 \mathrm{mg} / \mathrm{ml}$ 5-bromo-4-chloro-3indolyl phosphate toluidine salt (BCIP) and $75 \mathrm{mg} / \mathrm{ml}$ nitro blue tetrazolium chloride (NBT) solution (Sigma) with levamisole for the inhibition of endogenous phophatase activity, and mounted with Crystal mount (Biomeda). Negative control was same reactions omitting TdT from the labeling mix.

\section{Results}

\section{Morphological and biochemical changes in primary cortical culture}

Rat cortical neurons were cultured for 2 to 3 weeks and were characterized morphologically and biochemically. Under phase contrast microscopy, cortical neurons at DIV5 (5 days in vitro) extended prominent neurites (Figure $1 \mathrm{~A})$. Few synapses were present at DIV5, but many nascent synaptic junctions were observed. At DIV10, cortical neurons had an elaborate plexus of axons, dendrites, and synapses, indicating neuronal maturation (Figure 1B). At DIV20, the number of cortical neurons was reduced and the prominent destruction of associated neurites was evident. Even the remaining neurites had narrow and rough shapes (Figure $1 \mathrm{C}$ ). In addition, cortical cultures were contaminated with astrocytes at DIV15. Cultures did not contain any neurons but only astrocytosis at DIV30 (data not shown).

For biochemical evaluation, we examined the expressions of synaptic proteins such as synaptophysin and syntaxin by Western blotting. Synaptophysin is a structural protein of synaptic vesicles and syntaxin is a marker of the presynaptic membrane (Elferink and Scheller, 1995). Synaptophysin and syntaxin were maximally expressed around DIV10 and then gradually decreased while the level of actin, a loading control marker, was constant (Figure 1D). The levels of these synaptic proteins were nearly undetectable from DIV20.

\section{Characterization of neuronal loss during cultivation by TUNEL assay}

To characterize neuronal loss, TUNEL (Terminal deoxynucleotidyl transferase-mediated dUTP nickend labeling) assay was performed from DIV15. Interestingly, neurons at DIV15 were $60 \%$ TUNEL positive, although the numbers of attached neurons rarely decreased and the morphologies of neurites and synapses were relatively intact by phase contrast microscopy (Figure $2 \mathrm{~B}$ and $2 \mathrm{C}$ ). Contaminating non-neuronal cells were not detected by TUNEL staining.
A

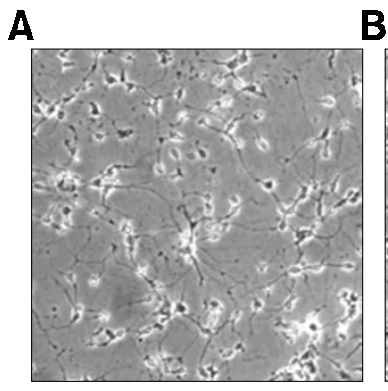

B

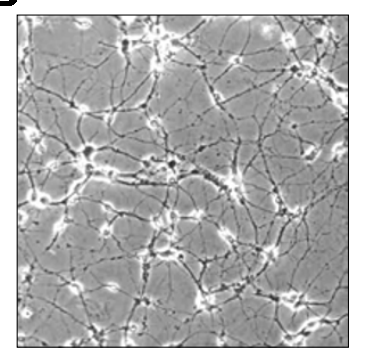

C

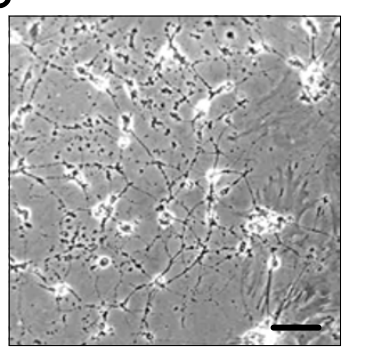

D

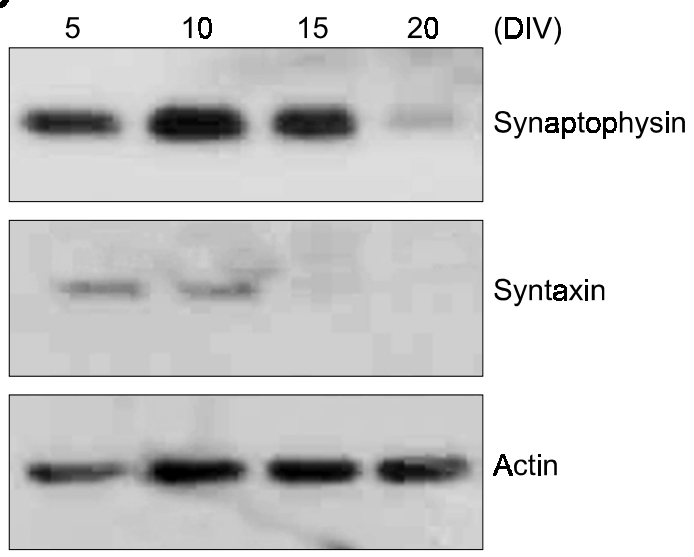

Figure 1. The morphology of rat embryonic cortical neurons is changed during cultivaiton. (A) Neurons at DIV5. (B) Neurons at DIV10. (C) Neurons at DIV20. Regional proliferation of non-neuronal cells is shown in left lower side (Bars $=100 \mu \mathrm{m}$ ). (D) Expressions of synaptic proteins are decreased during cultivation. Cells at each DIV were analyzed by western blotting for the synaptic proteins, synaptophysin and syntaxin, which are markers for synaptic vesicles or presynaptic membrane. The level of actin represents quantitative control of western blotting. All experiments were repeated at least three times and representative data are shown. 
A

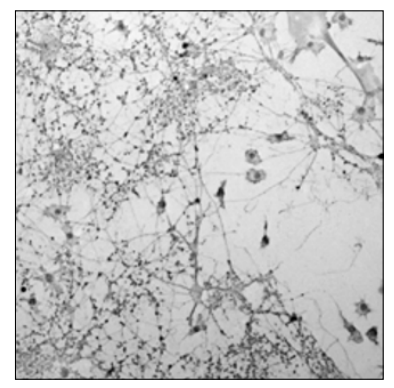

B

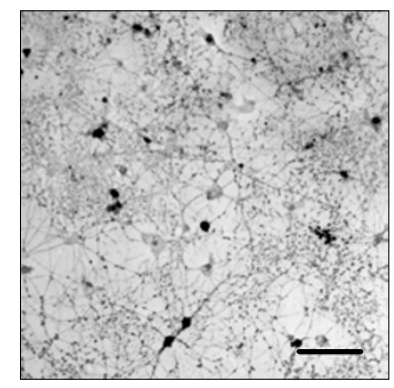

C

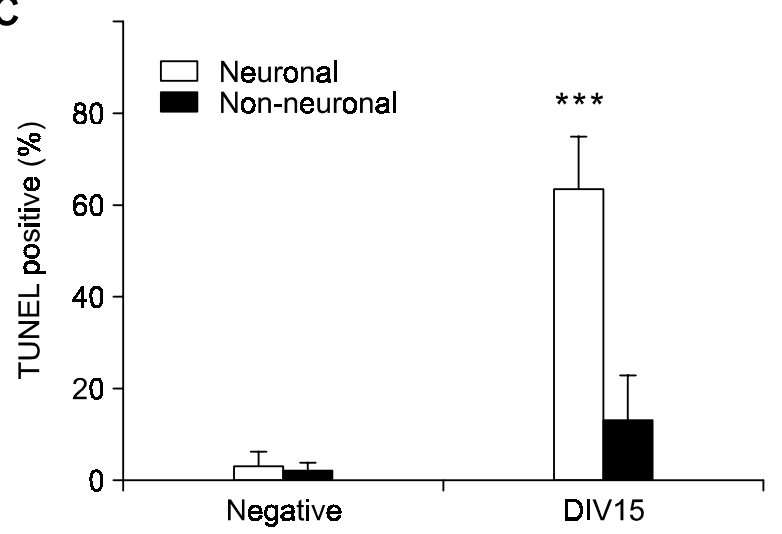

Figure 2. Long-term cultured primary cells show the neuron specific destruction or death. Terminal deoxynucleotidyl transferase-mediated dUTP nick-end labeling (TUNEL) assays were performed at DIV15 cells to identify quantity of neuronal cell death. (A) Cells at DIV15 without terminal deoxynucleotidyl transferase (TdT) incubation. (B) Nuclei of cells at DIV15 are stained by TUNEL method and the morphology of stained cells is neuronal. (C) Statistical analysis of TUNEL staining in cells at DIV15 is presented. Neuronal and non-neuronal cells were distinguished by morphological criteria, the presence/absence of neurites, and 'Negative' represent cells incubated without TdT. Several hundred neurons were scored for each condition at least three times and the results are given as mean \pm standard error of the mean (SEM) and statistical significance is indicated as ${ }^{* * *} P<0.001$ by Student's $t$-test $($ Bars $=100 \mu \mathrm{m})$.

A

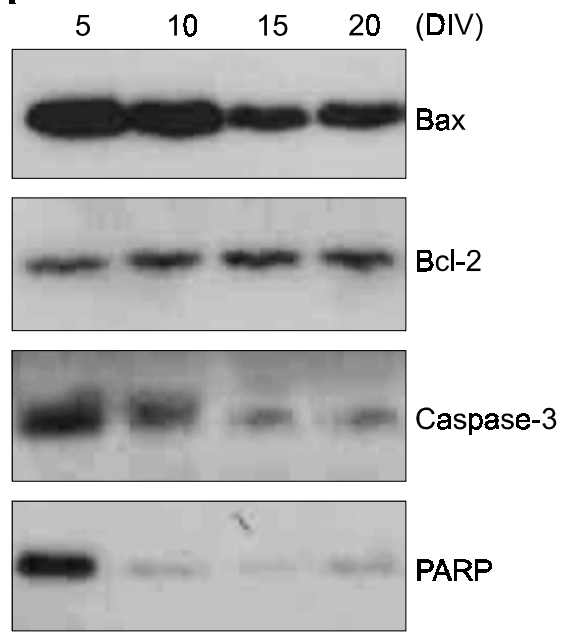

B

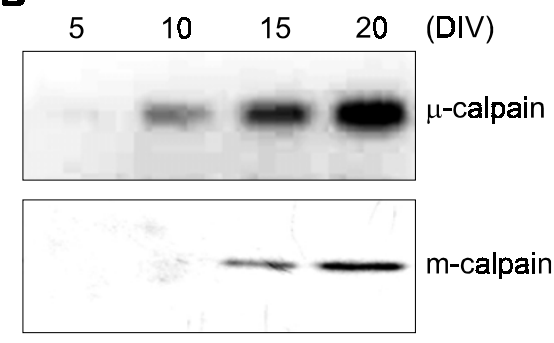

Figure 3. The death of long-term cultured neuron is associated with accumulation of calpain proteins. Levels of several apoptotic molecules are analyzed in cultured neuron by immunoblotting at indicated times. (A) Expressions of bax, bcl-2, procaspase-3, and PARP (B) The levels of $\mu$-calpain and $m$-calpain. All experiments were repeated at least three times and representative data are shown.

\section{Patterns of apoptotic molecules during cultivation} To evaluate the mechanism of neuronal death in long-term cultures, we performed western blotting for several apoptotic molecules, including bax, bcl-2, procaspase-3, and PARP (Figure 3A). The level of Bax (a pro-apoptotic protein) decreased from DIV15, but the level of bcl-2 (an anti-apoptotic protein) remained constant during cultivation. Procaspase-3 levels were also decreased from DIV15, but PARP (a target of caspase-3), decreased from DIV10 before caspase- 3 reduction.
The level of members of the calpain family of cysteine proteases were examined and compared with caspase- 3 . Interestingly, the levels of $\mu$ - and $\mathrm{m}$-calpain increased from DIV10 (Figure 3B). These findings agree with the observed decrease in Bax and with the early cleavage of PARP, which preceded caspase- 3 processing, because Bax, caspase-3, and PARP are proteolytically processed by calpains (Kobayashi et al., 1990; Shah et al., 1996; Wood et al., 1998; McGinnis et al., 1999b). Thus, we examined the levels of $\mu$ - and $m$-calpain. 
A
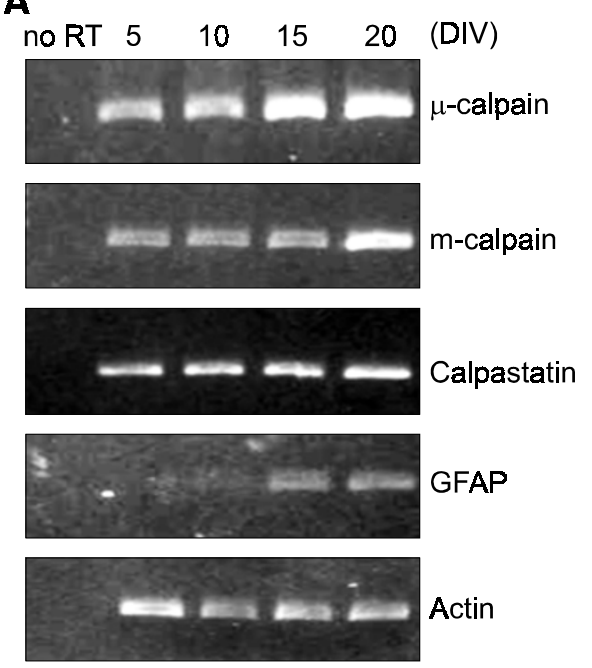

C
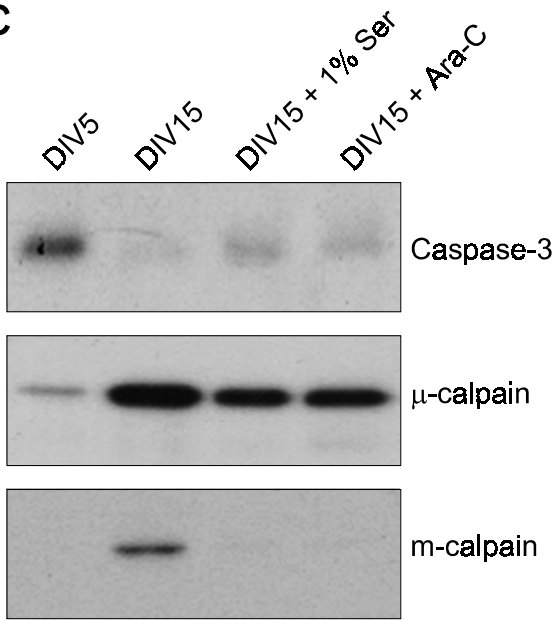

\section{The accumulation and activation of} calpains during cultivation

To study how the levels of calpain proteins were increased, we performed reverse transcription-polymerase chain reaction (RT-PCR). Both of $\mu$ - and $\mathrm{m}$-calpains were increased from DIV15 but the level of calpastatin (an endogenous calpain inhibitor) was unchanged (Figure 4A). Therefore, the up-regulation of these two calpains at the protein levels seems to be the result of the up-regulation of the transcription.

Next, we investigated which cultured cell types showed calpain accumulations, because astrocyte contamination and subsequent reactive astrocytosis were evident from DIV15. Initially, we examined the level of glial-fibrillary acidic protein (GFAP) (an astrocyte marker) by RT-PCR and as shown Figure 4A, the level of GFAP increased from DIV15. To confirm where the accumulation and activation of calpains originated from, neurons or glia, we repressed glial or non-neuronal populations by low serum cultivation and intermittent treatment with cytosine arbinoside (Ara-C) (Figure 4B). Low serum (1\%) or intermittent Ara-C treatment was started from DIV5 and Ara-C was treated at $5 \mu \mathrm{g} / \mathrm{ml}$ every 5 days to minimize the toxicity and the other effects of Ara-C. As expected, low serum and Ara-C treatment repressed GFAP up-regulation (Figure 4B) and morphological astrocytosis. However, $\mu$ - and $m$-calpain were not reduced by glial suppression. These results suggest that the accumulations of $\mu$-calpain and $\mathrm{m}$-calpain occur in neuronal cells, but not in glial cells.

To measure the activities of $\mu$ - and m-calpain, western blotting was performed for caspase-3, $\mu$ calpain, m-calpain, and fodrin (Figure 4C and 4D). Cells at DIV15 under conditions of glial suppression showed lower levels of caspase- 3 than cells at DIV5. In contrast to caspase- 3 , the levels of $\mu$-calpain at 
A

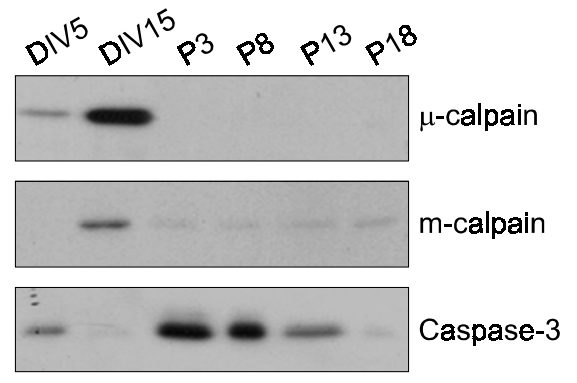

C

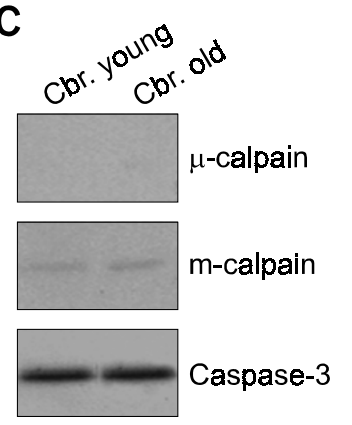

B

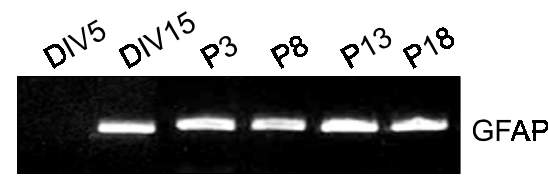

Figure 5. Accumulation and activation of calpains are shown only in long-term cultured neuron, but not in postnatal or aged cortical tissue. 'young' and 'old' shows each rat (Sprague-Dawley) cortical tissue of 6 and 29 months. (A) Levels of calpains and caspase-3 are analyzed by western blotting in cultured neuron and postnatal cortical tissues. (B) RTPCR analysis of GFAP levels. (C) Levels of calpains and caspase-3 are analyzed by western blotting in young ( 6 months) and old (29 months) rat cortices. (D) Cleavage of fodrin in postnatal, young, and old rat cortex, and in cultured neurons. All experiments were repeated at least three times and representative data are shown.
DIV15 were reduced after $1 \%$ serum and intermittent Ara-C treatment, but these were still higher than at DIV5. Interestingly, m-calpain showed a dramatic reduction after glial suppression by $1 \%$ serum and intermittent Ara-C treatment. To confirm the correlation between the activity and accumulation of calpains, we tested the cleavage of fodrin (a substrate of both calpains) (Figure 4D). Fodrin (also called $\alpha$ ll-spectrin) is a cytoskeletal protein of $280 \mathrm{kD}$ that is cleaved to 150 and $145 \mathrm{kD}$ fragments by calpains or to 150 and $120 \mathrm{kD}$ by activated caspase-3 (Wang, 2000). We initially found that even in neurons at DIV5 calpain-mediated fodrin cleavage was increased, compared with cortex tissue at 18th embryonic day. Cells at DIV15 showed reduced calpain cleavage bands (145 and $150 \mathrm{kD}$ ) but cells with glial suppression at DIV15 showed the sustained activation of calpains (145 and $150 \mathrm{kD}$ cleavage bands) together with the $120 \mathrm{kD}$ caspase- 3 cleavage band. These data shows that only primary neurons at DIV15, but not glia, sustained activation of calpains and increased activation of caspase- 3 . The purity of neuronal cells were checked by Western blot for NeuN neuronal marker (Mullen et al., 1992).

\section{Calpain activities during postnatal development and neuronal loss during long-term culture}

To determine whether the accumulation and activation of calpains and neuronal death after long-term culture are caused by developmental apoptosis of central nervous systems, we compared cultured neurons with the postnatal cortical tissues from rat matched for the duration in vitro culture. The in vitro culture and in vivo tissue samples showed that procaspase-3 processing was more prominent in postnatal cortical tissues (Figure 5A). Next, we compared the levels of both calpains between in vivo and in vitro conditions. First, all samples were examined using RT-PCR for GFAP, because, as shown by Figure $4 \mathrm{~A}$ and $4 \mathrm{C}$, glial cells including astrocytes might contain endogenous basal levels of calpains. All samples except cells at DIV5 were found to contain GFAP (Figure 5B). However, postnatal cortical tissues did not express $\mu$ - or $\mathrm{m}$-calpain (Figure 5C), but neurons at DIV15 contained high levels of calpains. We checked the activity of calpains by examining fodrin cleavage. As shown in Figure 5D, fodrin protein was not cleaved in postnatal cortical tissues as much as in cultured neurons.

\section{Involvement of p35 processing in long-term neuron culture}

To define the mechanism of calpain-induced death in long-term culture more precisely, we focused on the processing of $p 35$ protein. The processing of p35 to p25 is associated with the phosphorylation of neuronspecific cyclin-dependent kinase 5 (CDK5) and the subsequent pathologic phosphorylation of tau protein, which is an important aspect of tauopathy in Alzheimer's disease (Maccioni et al., 2001). Re- 
cently, it was found that the cleavage of p35 into p25 could occur by calpain activation (Patrick et al., 1999; Lee et al., 2000). Figure 6A shows the processing of p35 in embryonic 18 day cortical tissue and in vitro cultures at DIV5 and DIV15. In parallel with the fodrin western blot results in Figure $5 \mathrm{D}$, the cleavage of p35 to p25 started to occur on DIV5. Untreated primary cells at DIV15 did not show p35 band because the number of NeuN-positive neurons in the cell population was relatively low. But, the cells treated with $1 \%$ serum or with Ara-C at DIV15 displayed p35 to p25 conversion more so than untreated cells. In particular, cells treated with intermittent Ara-C at DIV15 showed a p25 band only, indicating full conversion of p35.

Next, we examined the p35 levels with respect to developmental and aging processes (Figure 6B). Postnatal cortical samples up to postnatal 13 day (P13) showed p25-cleaved band, but not at P18 as observed in vitro at DIV20. In cerebral cortex of young (7 months) and old (29 months) rats, p25 product was not detected. These results suggest that the neuronal loss during long-term culture was not the result of developmental or aging processes, although transient cleavage of p35 was observed in postnatal tissues, but it might be associated with neurodegeneration via calpain and p35.
A

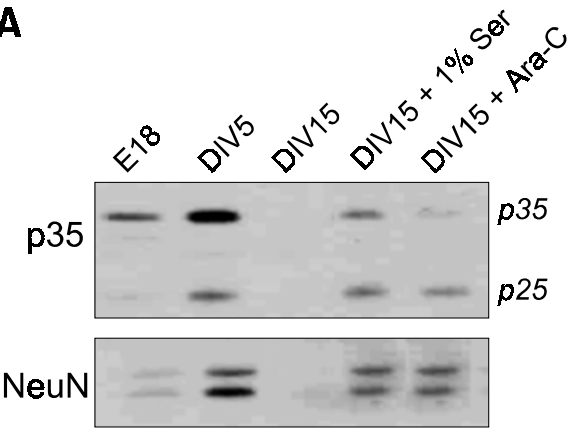

B

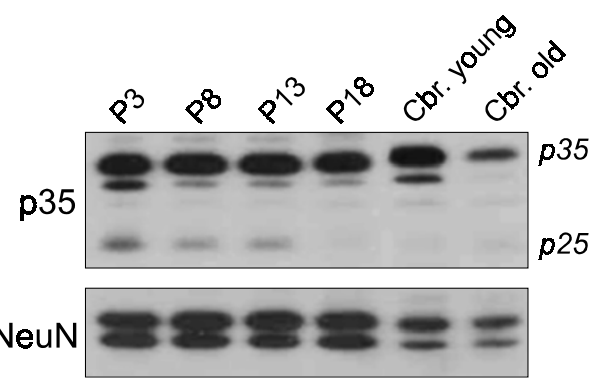

Figure 6. p35 converts to p25 in long-term primary neuronal culture, but not in postnatal or aged cortical tissue. Conversion of p35 to p25 was examined in neuronal culture and postnatal (P3-P18), young (7 months), and old (29 months) cerebral cortex of rat by western blotting. (A) p35 processing into p25 are shown in cells at DIV5 and DIV15 with $1 \%$ serum and Ara-C treatments. NeuN neuronal marker represents density of neurons. (B) p35 during postnatal periods is transiently processed into p25, and p35 in young and old cerebrum is not processed into p25. All experiments were repeated at least three times and representative data are shown.

A

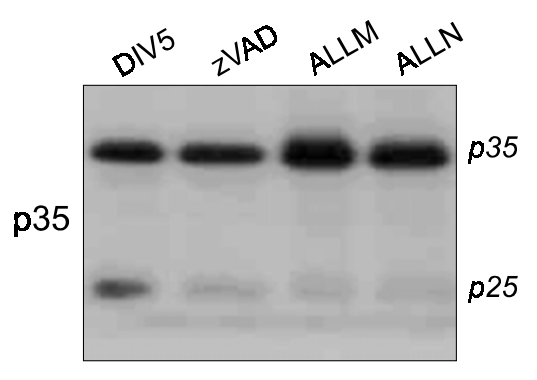

B

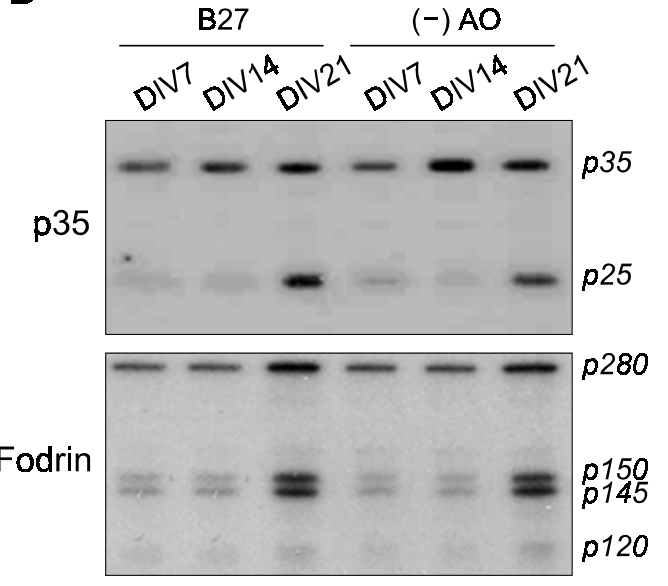

Figure 7. Caspase inhibitor or calpain inhibitors block conversion of p35 to p25. (A) Western blotting of p35 was performed at DIV5 after treatment with $100 \mu \mathrm{M}$ zVAD-fmk, $20 \mu \mathrm{M}$ ALLN and $20 \mu \mathrm{M}$ ALLM. (B) Culture of primary neuron using Neurobasal medium with B27 or B27AO (without antioxidants) also shows the conversion of p35 to p25 despite a delayed manner. All experiments were repeated at least three times and representative data are shown. 


\section{The effects of calpain inhibitors on the cleavage of $\mathrm{p} 35$}

The above data demonstrates that the death of long-term cultivated neurons may be associated with the accumulation and activation of calpains. Thus, we examined whether calpain inhibition blocks the processing of p35 (Figure 7A). We measured the initial processing of $p 35$ at DIV5, since it has been reported that ALLM (N-Acetyl-Leu-Leu-Met-CHO) and ALLN (N-Acetyl-Leu-Leu-Nle-CHO) are toxic to some mammalian cells (Fallis et al., 1999; An et al., 2000). ALLN and ALLM at $20 \mu \mathrm{M}$ were not toxic to DIV5 neurons (tested by trypan blue exclusion assay) and blocked the calpain activation via western blot for Fodrin (data not shown). In parallel, zVADfmk (z-Val-Ala-AspOMe- $\mathrm{CH}_{2} \mathrm{~F}$, a pancaspase inhibitor) was also tested. It was found that $z V A D-f m k$ and the two calpain inhibitors blocked p35 to p25 processing by western blotting of neurons at DIV5.

Finally, we examined whether cultured neurons in serum-free Neurobasal ${ }^{\mathrm{TM}}$ media (GIBCO, USA) exhibit p35 - p25 processing (Figure 7B). These media were supplemented with $B 27^{\mathrm{TM}}$ or $\mathrm{B} 27$ minus $A O^{\mathrm{TM}}$ (B27 supplement without anti-oxidants), since antioxidants such as Vitamin E, Vitamin A and glutathione have neuroprotective effects. Cultured neurons at DIV21 showed a cleavage band of p25 under both conditions. Moreover, the cleavage of fodrin into 145 $\mathrm{kD}$ or $150 \mathrm{kD}$ coincided with the cleavage of p35 protein indicating that calpains were also activated in Neurobasal ${ }^{\mathrm{TM}}$ media with some delays compared to other serum-supplemented media

\section{Discussion}

The aim of this study was to investigate the mechanism of neuronal loss in long-term primary neuronal cultures with respect to neuronal development, aging, and neurodegeneration. During the cultivation of primary neurons, neuronal loss and neurite degeneration were observed by morphological analysis and the levels of synaptic vesicle-associated proteins, including synaptophysin and syntaxin, were reduced. Moreover, at DIV15 when neuronal loss was apparent, $60 \%$ of remaining or morphologically wellattached neurons were TUNEL-positive, and this apoptotic phenomenon occurred in parallel with a reduction in procaspase-3. However, PARP (a target substrate of caspase-3) was initially cleaved before the activation of caspase- 3 , suggesting the downregulation of PARP protein or the cleavage by other proteases. Interestingly, Bax protein was reduced whereas Bcl-2 levels remained constant. Therefore, we examined calpain proteases, rather than caspase- 3 , because it has been reported to cause cell death through Bax and PARP cleavage (Wang, 2000).

Thus, we hypothesized that $\mu$-calpain and $\mathrm{m}$ calpain in long-term culture could cleave bax, procaspase-3, and PARP, but not bcl-2. The levels of these two calpains were very low at DIV5, but they both accumulated from DIV10. This is a rare phenomenon in vitro cell culture conditions, although some in vivo experiments found that $\mu$ calpain and m-calpain were abnormally high in the brains of Alzheimer's disease patients (Nilsson et al., 1990; Nixon et al., 1994). Moreover, in Parkinson's disease, $\mathrm{m}$-calpain is quantitatively upregulated in the substantia nigra and locus coeruleus (MouattPrigent et al., 1996). Thus, we investigated whether the protein levels of these two calpains in primary neuronal culture are increased, and compared their RNA levels. RT-PCR showed that the mRNA levels of $\mu$-calpain and $m$-calpain were increased during long-term culture of primary neurons, but the level of calpastatin (an endogenous inhibitor of calpain) was unchanged. These data showed that net ratio of calpain to calpastatin was increased, and suggest that neuronal loss in long-term culture may be associated with increased calpain expression. Additionally, we measured the activities of calpains by following the cleavage of fodrin (a cytoskeletal protein), which is a common target substrate of $\mu$-calpain or m-calpain. The 150 and $145 \mathrm{kD}$ cleavage products by calpains and a $120 \mathrm{kD}$ product by caspase- 3 are generated. As compared with the 18th embryonic day (E18), cells at DIV5 showed a calpain-specific cleavage bands, but cells at DIV15 showed a different pattern of reduced cleavage pattern, which was not correlated with the microscopic features of neuronal cell death. We believe that these results might be due to glial or nonneuronal cell contamination, and thus we tried to repress the glial population and isolate pure neuronal cells.

Glial suppression with low serum or intermittent Ara-C treatment did not change the mRNA levels of $\mu$ - and $\mathrm{m}$-calpain, however, interestingly, reduced the protein levels of the two calpains. This may indicate that the calpains in isolated neuronal or glia-suppressed cultures are more enzymatically active as confirmed by the cleavage pattern of fodrin. Cells cultured with glial suppression at DIV15 not only showed calpain cleavage activity, but also showed the caspase-3 $120 \mathrm{kD}$ cleavage product, whereas untreated cells did not. Surprisingly, cultured neurons showed strong cleavage of fodrin from DIV5. In addition, this synergistic effect of calpains and caspase-3 during long-term cultivation of primary neurons has been reported in focal ischemia of rat cortex and in cell death by amyloid precursor protein (APP) in neuroblastoma cells (Blomgren et al., 2001; 
Kuwako et al., 2002). Thus, we conclude that neuronal loss by long-term culture occurs gradually and is associated with the initial activation and accumulation of calpains and the subsequent activation of caspase-3.

Cell death during normal development has been described for almost all neuronal cell types in central and peripheral nervous systems (Oppenheim, 1991; Vaux and Korsmeyer, 1999). Although the underlying molecular mechanisms are still not clear, several relationships have been unearthed. These suggest the importance of early neural cell death in postnatal neural development, which is supported by the dramatic phenotype changes when death is prevented by capase-3, caspase- 9 or Apaf1 gene disruption (Kuida et al., 1996; 1998; Honarpour et al., 2000). Also, it has been reported that caspase- 3 is upregulated and activated in the perinatal developing brains (Mooney and Miller, 2000; Shimohama et al., 2001). Thus, neuronal loss due to long term embryonal cortex culture was compared with developmental cerebral cortex apoptosis, because primary neuronal culture is usually set up at 16-18 embryonal days and thus neuronal loss in culture might be a part of the developmental apoptosis. Therefore, we measured the levels of caspase- 3 and of the two calpains in the culture samples and in the tissue samples of matched postnatal days. We found that procaspase- 3 was gradually processed in vivo in rat cortex indicating developmental apoptosis, as reported previously. However, $\mu$-calpain and m-calpain were not detected in the in vivo cortex, but the long term culture (DIV15) showed caspase-3 processing and the accumulations of $\mu$ - and m-calpain simultaneously. These results suggest pathological apoptosis via the calpain system in long term cultures while developmental apoptosis in postnatal CNS. Moreover, no calpain activity was found in the postnatal cortex. So, we concluded that the neuronal loss by long-term cultivation does not comprise developmental apoptosis, since developmental apoptosis seems to be associated with caspase-3 activity, but not with activation of calpains.

Next, we investigated the association between long-term culture and neurodegeneration. The activation of calpains has been reported to feature in the pathogenesis of Alzheimer's disease (Huang and Wang, 2001). Two major pathological events are consistently identified in the Alzheimer's diseased brain, which involve amyloidopathy (amyloid plaque) and tauopathy (neurfibrillary tangle) (Hardy and Selkoe, 2002; Michaelis et al., 2002). In the etiology of tauopathy, the hyperphosphorylation of tau protein by CDK5 and by other kinases is an important step leading to the dissociation of microtubules and the formation of intracellular tau aggregates in a paired helical filament conformation. The activation of calpains would set in motion a cascade of calpainmediated p35 to p25 conversion, cdk5 activation, tau hyperphosphorylation and aggregation, neurofibrillary tangle formation, and ultimate neuronal death (Patrick et al., 1999). Thus, we tested for the conversion of p35 to p25 to determine whether the accumulation and activation of calpains in culture are associated with neurodegeneration, such as that in Alzheimer's disease. During long-term neuronal culture, p35 was found to be gradually processed into p25 in a calpain- dependent manner in parallel with the cleavage of fodrin. In particular, samples treated with intermittent Ara-C at DIV15 showed almost complete conversion of p35 to p25. In case of neuronal cells cultured in a serum-free medium (Neurobasal Media), the processing of p35 was also found to occur, despite delayed response than in serum-supplemented media. Furthermore, we compared the processing of p35 to p25 in the cerebral cortex tissues of perinatal (3-18 postnatal day), young ( 7 months), and old (29 months) rats. Perinatal cortex at 3-18 postnatal days showed an early and transient cleavage of p35 to p25, whereas cultured neuronal cells showed the strong cleavage product of p35. Moreover, immunoreactivity of p25 at 18 postnatal days was not observed, possibly because it reached the end of transient apoptosis for postnatal cortex development. Young and old cortices showed no p25 band, although the level of p35 protein was slightly reduced. Although the conversion of p35 to p25 occurred in the perinatal cortices, cultured neurons (i.e. glia suppressed cells) fully processed p35 to p25 as reported previously (Lee et al., 2000). Because of these differences, we believe that the neuronal loss during long-term culture bear more resemblance to neurodegeneration, as in Alzheimer's disease, than to developmental or aging processes.

Although our data did not show activation of calpains and p35 processing in aged cortex of SD (Sprague-Dawley) rats, several reports indicated these activation in aged brains (Kimura et al., 2003; Hinman et al., 2004; Bernath et al., 2006). In aged Wister rat brains, breakdown of cytoskeletal proteins such as fodrin and MAP-2 in neurons by calpains was elevated (Kimura et al., 2003) and, in aged monkey brains, activated microglia with calpain activation was observed (Hinman et al., 2004). However, these studies showed different modes of calpain activation depending on the cell types and degenerative regions. Moreover, as for the degree of the calpain activation, normal aged human brain did not show activated calpain as much as Alzheimer's diseased brain (Saito et al., 1993; Tsuji et al., 1998). From these, we concluded that the lack of cleavage 
of fodrin and p35 processing in aged cortex of SD rats might be due to the differences in strains and/or species as mentioned by Kimura et al. and less extent of normal aging than that of degenerative disorders. Furthermore, our data showed that aged SD rat brain exhibited decreased p35 levels with no p25 band rather than p35 processing into p25. These could be due to the decreased p35 expression or its degradation during the aging process. According to recent data, p35 can be degraded by proteasome in glutamatergic synapses when synaptic plasticity was increased by glutamate receptor agonists, NMDA or kinate, although this proteosomal regulation of $\mathrm{p} 35$ in aged brain needs to be examined further (Wei et al., 2005).

Many in vitro neurodegenerative model systems have been suggested for Alzheimer's and Parkinson's disease (Blum et al., 2001; Hardy and Selkoe, 2002), and have been used to examine the effects of neurotoxins like amyloid-beta peptides, apolipoprotein E, 6-hydroxydopamine (6-OHDA), and 1-methyl4-phenyl-1,2,3,6-tetrahydropyridine (MPTP), and to examine the effects of gene mutations or disruptions (Liu and Hong, 2005). However, these models have several shortcomings because; i) The effective levels of toxins required to cause degeneration is too high; ii) The pattern of cell death is usually uniform; iii) Cell death or degeneration occurs over a short period of time, which could mean acute neuronal injury, and because, iv) regional selectivity cannot be investigated as compared with in vivo models. Considering these problems, our long term neuronal culture can have some advantages as a model of neuronal degeneration since it does not require a neurotoxin treatment; and it can be used for studying culture methods. In fact, as mentioned above, increased calpain mRNA expression or protein accumulation cannot be effectively reproduced by in vitro neurotoxin treatment using neuron-like cell lines or primary cultures, because the proteolytic activation of calpains involves 'qualitative' regulation not a 'quantitative' change by RNA expression. However, in vivo studies reported high calpain immunoreactivity in many neurodegenerative disorders (the increase of total calpains), and the activation of calpain by cleavage or by autolytic-specific antibodies (the increase of activated calpain), suggesting both 'qualitative' and 'quantitative' regulation (Nilsson et al., 1990; Nixon et al., 1994; Tsuji et al., 1998). Our results demonstrate that primary longterm culture of the cortex itself is a good model system of neurodegeneration and the up-regulation (quantitative) and activation (quantitative) of calpain systems are involved in degeneration of primary neuronal culture. But further studies will be needed to examine other molecules, such as CDK5 and tau phosphorylation, involved in the pathogenesis of neurodegeneration.

\section{Acknowledgment}

We thank Dr. Kyungsook Ahn for her help in editing this manuscript. This study was supported by a grant of the Korean Health 21 R\&D Project, Ministry of Health and Welfare, Republic of Korea (02-PJ10- PG6-AG01-0010).

\section{References}

Aguzzi A, Brandner S, Marino S, Steinbach JP. Transgenic and knockout mice in the study of neurodegenerative diseases. J Mol Med 1996;74:111-26

An WG, Hwang SG, Trepel JB, Blagosklonny MV. Protease inhibitor-induced apoptosis: accumulation of p53, p21WAF1/ CIP1, and induction of apoptosis are independent markers of proteasome inhibition. Leukemia 2000;14:1276-83

Ando Y, Miyachi Y, Imamura S, Kannagi R, Murachi T. Purification and characterization of calpains from pig epidermis and their action on epidermal keratin. J Invest Dermatol 1988;90:26-30

Banker GA, Cowan WM. Rat hippocampal neurons in dispersed cell culture. Brain Res 1977:126:397-42

Bernath E, Kupina N, Liu MC, Hayes RL, Meegan C, Wang KK. Elevation of cytoskeletal protein breakdown in aged Wistar rat brain. Neurobiol Aging 2006;27:624-32

Blomgren K, Zhu C, Wang X, Karlsson JO, Leverin AL, Bahr $\mathrm{BA}$, Mallard C, Hagberg H. Synergistic activation of caspase-3 by $\mathrm{m}$-calpain after neonatal hypoxia-ischemia: A mechanism of "pathological apoptosis"? J Biol Chem 2001;276:10191-98.

Blum D, Torch S, Lambeng N, Nissou M, Benabid AL, Sadoul $\mathrm{R}$, Verna JM. Molecular pathways involved in the neurotoxicity of 6-ohda, dopamine and mptp: Contribution to the apoptotic theory in parkinson's disease. Prog Neurobiol 2001;65:135-72

Brewer GJ, Torricelli JR, Evege EK, Price PJ. Optimized survival of hippocampal neurons in b27-supplemented neurobasal, a new serum-free medium combination. J Neurosci Res 1993;35:567-76

Castagne V, Gautschi M, Lefevre K, Posada A, Clarke PG. Relationships between neuronal death and the cellular redox status. Focus on the developing nervous system. Prog Neurobiol 1999;59:397-23

Crispino M, Capano CP, Aiello A, lannetti E, Cupello A, Giuditta A. Messenger RNAs in synaptosomal fractions from rat brain. Mol Brain Res 2001;97:171-76

Czogalla A, Sikorski AF. Spectrin and calpain: a 'target' and a 'sniper' in the pathology of neuronal cells. Cell Mol Life Sci 2005;62:1913-24

Ecarnot-Laubriet A, Assem M, Poirson-Bichat F, Moisant M, Bernard C, Lecour S, Solary E, Rochette L, Teyssier JR. Stage-dependent activation of cell cycle and apoptosis mechanisms in the right ventricle by pressure overload. Biochim Biophys Acta 2002;1586:233-42| 
Elferink LA, Scheller RH. Synaptic vesicle proteins and regulated exocytosis. Prog Brain Res 1995:105:79-85

Evans MS, Collings MA, Brewer GJ. Electrophysiology of embryonic, adult and aged rat hippocampal neurons in serum-free culture. J Neurosci Methods 1998:79:37-46

Fallis LH, Richards E, O'Connor DJ, Zhong S, Hsieh JK, Packh Lu X. The biological response of MCF7 breast cancer cells to proteosome inhibition or gamma-radiation is unrelated level of p53 induction. Apoptosis 1999;4:99-107

Faulkner CB, Simecka JW, Davidson MK, Davis JK, Schoeb TR, Lindsey JR, Everson MP. Gene expression and production of tumor necrosis factor alpha, interleukin 1 , interleukin 6 , and gamma interferon in $\mathrm{c} 3 \mathrm{~h} / \mathrm{hen}$ and $\mathrm{c} 57 \mathrm{bl} / 6 \mathrm{n}$ mice in acute mycoplasma pulmonis disease. Infect Immun 1995;63:408490

Hardy J, Selkoe DJ. The amyloid hypothesis of alzheimer's disease: Progress and problems on the road to therapeutics. Science 2002;297:353-56

Hinman JD, Duce JA, Siman RA, Hollander W, Abraham CR. Activation of calpain-1 in myelin and microglia in the white matter of the aged rhesus monkey. J Neurochem. 2004; 89:430-41

Honarpour N, Du C, Richardson JA, Hammer RE, Wang X, Herz J. Adult apaf-1-deficient mice exhibit male infertility. Dev Biol 2000;218:248-58

Huang $Y$, Wang KK. The calpain family and human disease. Trends Mol Med 2001;7:355-62

Kim JA, Kim HL. Cleavage of purified neuronal clathrin assembly protein (CALM) by caspase 3 and calpain. Exp Mol Med 2001;33:245-50.

Kimura N, Tanemura K, Nakamura S, Takashima A, Ono F, Sakakibara I, Ishii Y, Kyuwa S, Yoshikawa Y. Age-related changes of Alzheimer's disease-associated proteins in cynomolgus monkey brains. Biochem Biophys Res Commun 2003;310:303-11

Kobayashi Y, Yamamoto K, Saido T, Kawasaki H, Oppenheim $\mathrm{JJ}$, Matsushima K. Identification of calcium-activated neutral protease as a processing enzyme of human interleukin 1 alpha. Proc Natl Acad Sci USA 1990;87:5548-52

Kuida K, Zheng TS, Na S, Kuan C, Yang D, Karasuyama H, Rakic P, Flavell RA. Decreased apoptosis in the brain and premature lethality in cpp32-deficient mice. Nature1996;384: 368-72

Kuida K, Haydar TF, Kuan CY, Gu Y, Taya C, Karasuyama H, Su MS, Rakic P, Flavell RA. Reduced apoptosis and cytochrome c-mediated caspase activation in mice lacking caspase 9. Cell 1998;94:325-37

Kuwako K, Nishimura I, Uetsuki T, Saido TC, Yoshikawa K. Activation of calpain in cultured neurons overexpressing alzheimer amyloid precursor protein. Mol Brain Res 2002; 107:166-75

Lee MS, Kwon YT, Li M, Peng J, Friedlander RM, Tsai LH. Neurotoxicity induces cleavage of p35 to p25 by calpain. Nature 2000;405:360-64

Litersky JM, Johnson GV. Phosphorylation of tau in situ: Inhibition of calcium-dependent proteolysis. J Neurochem
1995;65:903-11

Liu ML, Hong ST. Early phase of amyloid beta42-induced cytotoxicity in neuronal cells is associated with vacuole formation and enhancement of exocytosis. Exp Mol Med. 2005;37:559-66.

Maccioni RB, Otth C, Concha II, Munoz JP. The protein kinase cdk5. Structural aspects, roles in neurogenesis and involvement in alzheimer's pathology. Eur J Biochem 2001;268: 1518-27

Martin LJ, Al-Abdulla NA, Brambrink AM, Kirsch JR, Sieber FE, Portera-Cailliau C. Neurodegeneration in excitotoxicity, global cerebral ischemia, and target deprivation: A perspective on the contributions of apoptosis and necrosis. Brain Res Bull 1998a;46:281-309

Martin LJ, Furuta A, Blackstone CD. Ampa receptor protein in developing rat brain: Glutamate receptor-1 expression and localization change at regional, cellular, and subcellular levels with maturation. Neuroscience1998b;83:917-28

McGinnis KM, Whitton MM, Gnegy ME, Wang KK. Calcium/calmodulin-dependent protein kinase iv is cleaved by caspase- 3 and calpain in sh-sy5y human neuroblastoma cells undergoing apoptosis. J Biol Chem 1998;273:19993-20000

McGinnis KM, Gnegy ME, Park YH, Mukerjee N, Wang KK. Procaspase-3 and poly(adp)ribose polymerase (parp) are calpain substrates. Biochem Biophys Res Commun 1999a; 263:94-99

McGinnis KM, Gnegy ME, Wang KK, Endogenous bax translocation in sh-sy5y human neuroblastoma cells and cerebellar granule neurons undergoing apoptosis. J Neurochem 1999b;72:1899-06

Michaelis ML, Dobrowsky RT, Li G. Tau neurofibrillary pathology and microtubule stability. J Mol Neurosci 2002; 19:289-93

Mooney SM, Miller MW. Expression of bcl-2, bax, and caspase-3 in the brain of the developing rat. Dev Brain Res 2000;123:103-17

Morrison JH, Hof PR. Life and death of neurons in the aging brain. Science 1997;278:412-19

Mouatt-Prigent A, Karlsson JO, Agid Y, Hirsch EC. Increased $\mathrm{m}$-calpain expression in the mesencephalon of patients with parkinson's disease but not in other neurodegenerative disorders involving the mesencephalon: A role in nerve cell death? Neuroscience 1996;73:979-87

Mullen RJ, Buck CR, Smith AM. Neun, a neuronal specific nuclear protein in vertebrates. Development 1992;116:201-11

Nguyen MD, Mushynski WE, Julien JP. Cycling at the interface between neurodevelopment and neurodegeneration. Cell Death Differ 2002;9:1294-306

Nilsson E, Alafuzoff I, Blennow K, Blomgren K, Hall CM, Janson I, Karlsson I, Wallin A, Gottfries CG, Karlsson JO. Calpain and calpastatin in normal and Alzheimer-degenerated human brain tissue. Neurobiol Aging 1990;11:425-31

Nixon RA, Saito KI, Grynspan F, Griffin WR, Katayama S, Honda T, Mohan PS, Shea TB. Beermann M. Calciumactivated neutral proteinase (calpain) system in aging and Alzheimer's disease. Ann NY Acad Sci 1994;747:77-91 
Oppenheim RW. Cell death during development of the nervous system. Annu Rev Neurosci 1991;14:453-501

Patrick GN, Zukerberg L, Nikolic M, de la Monte S, Dikkes P, Tsai LH. Conversion of p35 to p25 deregulates cdk5 activity and promotes neurodegeneration. Nature 1999;402:615-22

Prasad SC, Thraves PJ, Kuettel MR, Srinivasarao GY, Dritschilo A, Soldatenkov VA. Apoptosis-associated proteolysis of vimentin in human prostate epithelial tumor cells. Biochem Biophys Res Commun 1998;249:332-38

Ray J, Peterson DA, Schinstine M, Gage FH. Proliferation, differentiation, and long-term culture of primary hippocampal neurons. Proc Natl Acad Sci USA 1993;90:3602-06

Ray SK, Matzelle DC, Wilford GG, Hogan EL, Banik NL. E-64-d prevents both calpain upregulation and apoptosis in the lesion and penumbra following spinal cord injury in rats. Brain Res 2000;867:80-89

Saito K, Elce JS, Hamos JE, Nixon RA. Widespread activation of calcium-activated neutral proteinase (calpain) in the brain in Alzheimer disease: a potential molecular basis for neuronal degeneration. Proc Natl Acad Sci USA 1993;90:2628-32

Shah GM, Shah RG, Poirier GG. Different cleavage pattern for poly(adp-ribose) polymerase during necrosis and apoptosis in hl-60 cells. Biochem Biophys Res Commun 1996;229:838-44

Shimohama S, Tanino H, Fujimoto S. Differential expression of rat brain caspase family proteins during development and aging. Biochem Biophys Res Commun 2001;289:1063-66

Silani V, Braga M, Ciammola A, Cardin V, Scarlato G. Motor neurones in culture as a model to study als. J Neurol 2000;247: 128-36

Tsuji T, Shimohama S, Kimura J, Shimizu K. M-calpain (calcium-activated neutral proteinase) in Alzheimer's disease brains. Neurosci Lett 1998;248:109-12

Vaux DL, Korsmeyer SJ. Cell death in development. Cell 1999;96:245-54

Wang KK. Calpain and caspase: Can you tell the difference? Trends Neurosci 2000;23:20-26

Wei FY, Tomizawa K, Ohshima T, Asada A, Saito T, Nguyen C, Bibb JA, Ishiguro K, Kulkarni AB, Pant HC, Mikoshiba K, Matsui $\mathrm{H}$, Hisanaga S. Control of cyclin-dependent kinase 5 (Cdk5) activity by glutamatergic regulation of p35 stability. J Neurochem 2005;93:502-12

Wood DE, Thomas A, Devi LA, Berman Y, Beavis RC, Reed $\mathrm{JC}$, Newcomb EW. Bax cleavage is mediated by calpain during drug-induced apoptosis. Oncogene 1998;17:1069-78

Xie C, Markesbery WR, Lovell MA. Survival of hippocampal and cortical neurons in a mixture of mem+ and b27supplemented neurobasal medium. Free Radic Biol Med 2000;28:665-72 